\title{
Descripción y análisis de la teoría sobre seguimiento y evaluación aplicable a las actuaciones cofinanciadas por Fondos Europeos*
}

Verónica Viñas**

\section{Introducción}

En la actualidad, el Ministerio de Trabajo, el Ministerio de Economía, todas las comunidades autónomas y numerosos ayuntamientos, entre otros organismos públicos y privados, reciben dinero de la Unión Europea para programas de desarrollo regional. Según la normativa comunitaria actual, es obligatorio evaluar esos programas. Sin embargo, existe un gran desconocimiento por parte de los responsables públicos sobre por qué hay que evaluar, qué hay que evaluar y cómo. Este artículo contiene una descripción y análisis de la teoría sobre el seguimiento y la evaluación aplicable a las actuaciones cofinanciadas con Fondos Europeos. Consta de cuatro apartados: (1) El primero justifica la necesidad y utilidad de establecer un sistema de seguimiento de las intervenciones públicas cofinanciadas por los Fondos Europeos, tanto desde el punto de vista normativo como del económico-presupuestario. (2) El segundo apartado es un marco general para comprender los objetivos del seguimiento y la evaluación, ya que explica la lógica del establecimiento de objetivos en las intervenciones comunitarias, y cómo se programan -en líneas generaleslas intervenciones cofinanciadas con Fondos Europeos. (3) En el tercero se expone la doctrina sobre seguimiento y evaluación de la Comisión Europea, reinterpretándola y explicándola para una mejor comprensión. Se hace hincapié en la distinción entre seguimiento y evaluación, las herramientas para el seguimiento y la evaluación, la aplicación de los indicadores de seguimiento y la transmisión electrónica de la información. El último epígrafe de este tercer apartado resalta los elementos clave para la puesta en práctica del Sistema de seguimiento continuo propuesto. (4) El cuarto aborda sucintamente un tema de gran preocupación en la actualidad: el uso de las evaluaciones. El contenido del artículo puede ayudar tanto a los responsables políticos y gestores que encargan las evaluaciones, como a los profesionales de la evaluación que tienen que llevarlas a cabo.

\section{Justificación de la necesidad de un sistema de seguimiento y evaluación de las intervenciones cofinanciadas por los Fondos Europeos}

La necesidad de diseñar un sistema continuo de seguimiento y evaluación de las acciones públicas que son cofinanciadas por los Fondos Europeos (Estructurales y de Cohesión) tiene una doble justificación: la primera, de tipo normativo o jurídico; la segunda, de carácter económico presupuestario. 


\subsection{Desde el punto de vista reglamentario}

El decimocuarto "considerando" del Reglamento Marco de los Fondos Estructurales vigente en la etapa de programación anterior (1994-1999) (Reglamento CEE No 2081/93) establecía “(...) que conviene reforzar la apreciación previa, el seguimiento y la evaluación posterior y establecer una mayor flexibilidad en la ejecución de las intervenciones estructurales comunitarias a fin de responder a las necesidades reales; que, en aras de la eficacia, conviene proceder a una apreciación detallada antes de comprometer recursos comunitarios para garantizar que producirán ventajas socioeconómicas relacionadas con los recursos movilizados (...)".

Las evaluaciones intermedias realizadas durante la segunda mitad de 1997 por todos los organismos españoles responsables de la coordinación de las actuaciones cofinanciadas por los Fondos Estructurales pusieron de manifiesto la importancia que entraña, para la Comisión Europea, mejorar la calidad de los sistemas regionales y nacionales de información sobre los resultados físicos e impactos de las intervenciones.

Esta voluntad de la Comisión ha recibido un nuevo impulso en los actuales reglamentos que regulan el funcionamiento de la tercera etapa de programación de los Fondos Estructurales (2000-2006). En concreto, el vigente Reglamento por el que se establecen disposiciones generales sobre los Fondos Estructurales (Reglamento CE No 1260/99) dispone en sus considerandos:

"(...) que una de las garantías de la eficacia de la acción de los Fondos Estructurales es un seguimiento eficaz; que es necesario mejorar el seguimiento (...)” (considerando 46).

“(...) que es fundamental para el seguimiento que existan indicadores e informes anuales de ejecución y que es necesario definirlos mejor para que reflejen de manera fiable el grado de ejecución de las intervenciones y la calidad de la programación" (considerando 49).

"(...) que la eficacia y los efectos de las actividades de los Fondos Estructurales dependen también de que se mejore y profundice la evaluación, y que es necesario precisar las responsabilidades de los Estados miembros y de la Comisión en la materia, asi como algunas reglas que garanticen la fiabilidad de la evaluación" (considerando 53).

"(...) que conviene evaluar las intervenciones con miras a la preparación, aprobación, revisión intermedia y valoración de los efectos de las mismas (...)" (considerando 54).

"(...) que la evaluación intermedia y el hecho de reservar una parte de los créditos permiten asignar créditos suplementarios en cada Estado miembro en función del resultado de las intervenciones (...)" (considerando 55).

El Reglamento desarrolla estos principios dedicando el Título IV a la "Eficacia de las intervenciones de los fondos", con once detallados artículos referidos al seguimiento, a la evaluación y a la reserva de eficacia. Estos artículos refuerzan con mayor claridad aún la necesidad de mejorar los actuales sistemas de seguimiento y evaluación.

Aunque no hay una única forma europea de realizar evaluaciones, por lo que no podemos generalizar desde el caso español, las evaluaciones intermedias de la aplicación en España de los objetivos 2, 3 y 5b (realizadas durante 1997), las evaluaciones intermedias avanzadas de los objetivos 2 y 3 (realizadas en los años 2000 y 1999, respectivamente) y la evaluación final del Objetivo 5b (realizada en el año 2000), pusieron de manifiesto que el sistema de información y evaluación no se encontraba adaptado a los requerimientos solicitados por la Comisión, especialmente en tres aspectos: cuantificación de objetivos, generación de indicadores físicos sobre el desarrollo de las distintas acciones y evaluación de impactos derivados de dichas intervenciones (LUUKKONEN, 2002: 81).

\subsection{Desde el punto de vista económico-presupuestario}

No obstante lo apuntado, la implantación de un sistema de seguimiento y evaluación de las actuaciones desarrolladas por cualquier administración pública no constituye tan sólo una obligación legal con posibles repercusiones económicas, sino también una oportunidad, de cara a mejorar la gestión de las intervenciones dirigidas a impulsar los cambios estructurales en el tejido económico, social o territorial.

En buena medida, el desarrollo de un sistema racional y estructurado de información sobre la gestión de una parte muy considerable de la política estructural de las administraciones públicas debe generar necesariamente repercusiones significativas sobre la mejora de la calidad de su gestión pública, así como sobre la cantidad, calidad y transparencia de la información disponible sobre estas cuestiones, aspectos cada día más demandados, tanto desde el punto de vista político como social.

El seguimiento y evaluación de las acciones públicas es, en definitiva, una excelente vía para compatibilizar la creciente demanda social de bienes y servicios públicos con las restricciones existentes para el incremento del gasto público, ya que se trata de una herramienta de análisis (pero, sobre todo, de gestión) cuyo fin último estriba en suministrar información relevante (tanto a técnicos y gestores como a los órganos políticos en los que radica en última instancia la capacidad de decisión) para mejorar la asignación de unos recursos escasos, es decir, para aumentar la eficiencia del gasto público.

No obstante, el fin último del Sistema de seguimiento continuo al que hace referencia este artículo, no es sólo cumplir con la nor- 
mativa y contribuir a mejorar la gestión, sino también facilitar el trabajo a todos los organismos de la administraciones públicas implicados en actuaciones cofinanciadas por Fondos Europeos.

\begin{tabular}{|c|}
\hline $\begin{array}{c}\text { Recuadro } 1 \\
\text { Justificación de la necesidad de un Sistema de } \\
\text { seguimiento de las intervenciones públicas cofinanciadas } \\
\text { por los Fondos Europeos }\end{array}$ \\
\hline $\begin{array}{l}\text { El diseño y la aplicación de un Sistema de seguimiento continuo en } \\
\text { las administraciones públicas se justifican principalmente por las } \\
\text { siguientes razones: }\end{array}$ \\
\hline $\begin{array}{l}\checkmark \text { Cumplir con la normativa europea y regional vigente. } \\
\checkmark \text { Mejorar la programación y gestión de las intervenciones. } \\
\checkmark \text { Facilitar el trabajo de los organismos implicados. }\end{array}$ \\
\hline
\end{tabular}

\section{Las intervenciones comunitarias}

\subsection{La lógica general de las intervenciones comunitarias}

Una parte fundamental de la preparación de los programas de los Fondos Estructurales es el establecimiento de objetivos y la asignación de los recursos entre las diversas intervenciones con vistas a una consecución óptima de los objetivos. Existe una relación lógica entre las decisiones de asignación y los objetivos, que puede presentarse desde el nivel superior al inferior o desde el nivel inferior al superior. En la práctica, la programación implica la alternancia de estas dos perspectivas:
- Desde el nivel superior al inferior: todas las intervenciones se programan en un contexto específico con relación a un objetivo global establecido. Dicho objetivo constituye la base de la estrategia de la intervención y origina un cierto número de objetivos específicos que, de forma general, se corresponden con las áreas prioritarias. A su vez, cada objetivo específico se lleva a cabo a través de medidas. Estas últimas permiten la consecución de los objetivos operativos.

- Desde el nivel inferior al superior:

> Las medidas o actuaciones son llevadas a cabo por administraciones, organismos o agentes económicos que utilizan diversos medios o recursos, de índole financiera, humana, técnica u organizativa.

$>$ El gasto real origina una serie de realizaciones físicas (kilómetros de carretera construidos, número de puestos de formación ofertados, etc.), que reflejan los progresos de la ejecución de la medida.

Los resultados son los efectos inmediatos sobre los beneficiarios directos de las acciones financiadas (reducción del tiempo de transporte o de los costes de transporte, número de personas formadas, etc.).

> Estos resultados ayudan a conseguir los impactos, es decir, la consecución de los objetivos globales del programa. Los impactos pueden incluir, por ejemplo, el aumento del trafico de mercancías, una mejor adaptación de los trabajadores a las exigencias del mercado laboral o hacer referencia a la creación de empleo neto.

En el Esquema 1 se presenta la secuencia lógica de la intervención comunitaria.

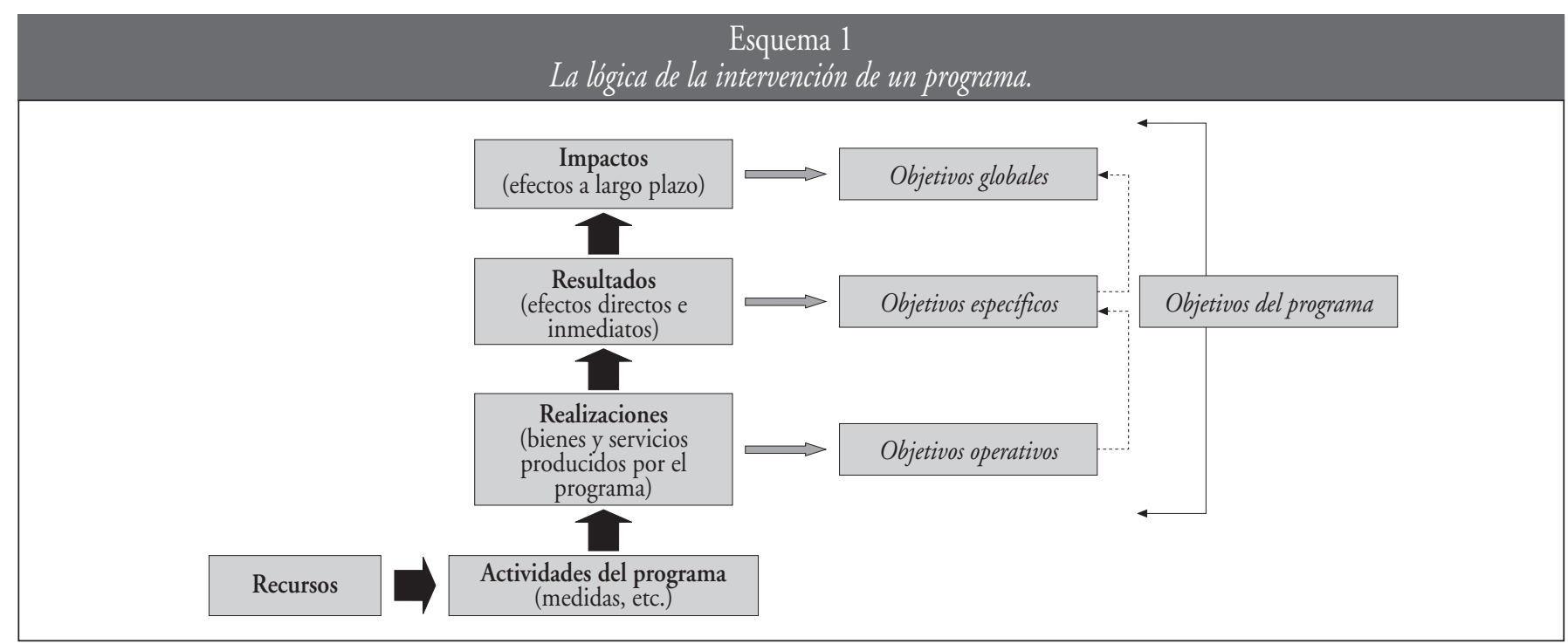

Fuente: Comisión Europea, 1999b:6. 
El esquema muestra cómo los recursos del programa están relacionados tanto con las realizaciones como con la consecución de los resultados y los impactos. Asimismo, presenta los medios a través de los cuales el programa alcanza sus objetivos operativos, específicos y globales. Este encadenamiento de elementos se basa en un esquema clásico en política económica, que relaciona agentes con instrumentos y fines.

En general:

- Los objetivos operativos se plasman en términos de realizaciones (por ejemplo, oferta de cursos de formación profesional a los desempleados de larga duración).

- Los objetivos específicos se plasman en términos de resultados (por ejemplo, aumento de las posibilidades de reincorporación al mercado laboral de los parados de larga duración).

- Los objetivos globales se plasman en términos del impacto del programa (por ejemplo, reducción del desempleo entre los desempleados de larga duración).

Recuadro 2
La lógica de la programación comunitaria
Es decir, en un plano conceptual puede utilizarse ese esquema para
comprender mejor la lógica de la programación de las intervenciones
comunitarias:
- Los diferentes organismos gestores realizan actividades utilizando
recursos o medios (financieros, humanos, técnicos, materiales, etc.).
Las actividades originan una serie de realizaciones (bienes ylo servicios
producidos).
La utilización de esos bienes o servicios permite obtener resultados o
efectos directos o inmediatos (que se corresponden con los objetivos
especificos).
Estos resultados deben, en su conjunto, ayudar a obtener un impacto
respecto al objetivo global (o de largo plazo) de las intervenciones.

Esta lógica es la que se ha representado gráficamente en el Esquema 1.

Conocer el grado de consecución de los resultados permite juzgar la calidad o el rendimiento de las realizaciones. Es decir, los objetivos específicos son el punto de referencia principal para los gestores responsables de la intervención. El éxito o el fracaso deberán medirse, sobre todo, en relación con este objetivo.

La programación se realiza, o debería realizarse, a partir de cierto número de hipótesis que la experiencia nos presenta como razonables, y que permiten esperar que determinadas realizaciones produzcan determinados resultados, los cuales, a su vez, permitirán obtener un impacto sobre la problemática. Pero esta lógica no es mecánica o automática. Puede estar condicionada por las propias características de las acciones, por otros factores no controlables o por la coyuntura económica. Ahí radica el interés de comprobar en el momento del seguimiento y-sobre todo- en las evaluaciones, las relaciones de causalidad entre realizaciones y resultados, y entre resultados e impactos.

Veamos, a continuación, algunos ejemplos ilustrativos, referidos a tres programas diferentes: uno de infraestructura, otro relacionado con los recursos humanos y el tercero relacionado con el apoyo al sector productivo.

\begin{tabular}{|l|l|}
\hline \multicolumn{2}{|c|}{ Cuadro 1} \\
\multicolumn{2}{|c|}{ La lógica de un proyecto de infraestructura } \\
\hline Realización & - Una nueva autopista. \\
\hline Resultados & $\begin{array}{l}\text { - Reducir el tiempo de recorrido. } \\
\text { - Reducir los costes de transporte. } \\
\text { - Mejorar la seguridad. }\end{array}$ \\
\hline Impactos & $\begin{array}{l}\text { - Incrementar los flujos interregionales de personas } \\
\text { - Inercancías. }\end{array}$ \\
& - Incrementar las actividades socioeconómicas. \\
\hline
\end{tabular}

Fuente: Elaboración propia a partir de: Comisión Europea, 1995:8.

\begin{tabular}{|l|l|}
\hline \multicolumn{2}{|c|}{ Cuadro 2} \\
\hline \multicolumn{1}{|c|}{$\begin{array}{c}\text { Lógica de un proyecto relacionado } \\
\text { con los recursos humanos }\end{array}$} \\
\hline Realización & • Cursos de formación. \\
\hline Resultados & $\begin{array}{l}\text { - Conseguir la finalización y superación de las } \\
\text { acciones de formación por parte de los } \\
\text { beneficiarios. } \\
\text { - Conseguir unos perfiles mejor adaptados a las } \\
\text { necesidades del mercado laboral. }\end{array}$ \\
\hline Impactos & - Incrementar las posibilidades de empleo. \\
\hline
\end{tabular}

Fuente: Elaboración propia a partir de: Comisión Europea, 1995:8.

\begin{tabular}{|c|c|}
\hline \multicolumn{2}{|c|}{$\begin{array}{c}\text { Cuadro } 3 \\
\text { La lógica de un programa de apoyo } \\
\text { al sector productivo }\end{array}$} \\
\hline Realización & - Un nuevo palacio de congresos. \\
\hline Resultados & $\begin{array}{l}\text { - Celebrar seminarios, conferencias, congresos y } \\
\text { encuentros empresariales. } \\
\text { - Mejorar los conocimientos y las informaciones } \\
\text { de que disponen los empresarios locales y } \\
\text { regionales. } \\
\text { - Facilitar los intercambios y la cooperación entre } \\
\text { estos empresarios. }\end{array}$ \\
\hline Impactos & - Contribuir al desarrollo de la región. \\
\hline
\end{tabular}

Fuente: Elaboración propia a partir de: Comisión Europea, 1995:8/9. 


\subsection{Programación de las intervenciones de los Fondos Europeos}

La intervención de los Fondos Europeos respeta la lógica de las intervenciones descrita en el apartado anterior, sujetándose a las restricciones derivadas de la aplicación de los principios de programación de los Fondos Europeos: marcos comunitarios de apoyo (MCA), programas operativos (P.O.), documentos únicos de programación (DOCUP) y complementos de programas. Los MCA presentan una serie de prioridades que se aplican a través de los P.O. A su vez, cada P.O. incluye una serie de prioridades compuestas de medidas plurianuales. Los DOCUP tienen una estructura más sencilla, formada por elementos incluidos tanto en los MCA como en los P.O. La reforma de los Fondos Estructurales vigente a partir del año 2000 introduce un nuevo concepto en el sistema de programación: el complemento de pro- grama. La principal consecuencia de esta novedad es que el Estado miembro pasa a ser competente en determinar el contenido del programa a nivel de la medida y de cuantificar los objetivos asociados. Tal como se muestra en el siguiente esquema, cada nivel de programación (MCA, P.O., etc.) está sujeto a la misma clasificación de objetivos. El objetivo global del nivel inferior corresponde al objetivo específico del nivel superior y, a la inversa, el objetivo específico del nivel superior corresponde al objetivo global del nivel inferior. No obstante, los objetivos operativos sólo existen en el nivel de las realizaciones físicas.

El siguiente esquema es el que expone la Comisión Europea para presentar la relación entre los diversos niveles de programación (MCA, P.O. y Complemento de Programa o DOCUP y Complemento de Programa). Sin embargo, es necesario aclarar que los documentos de programación que se han redactado han encontrado dificultades en su aplicación práctica.

\section{Esquema 2 \\ Relación entre los niveles y los efectos de la intervenciones}

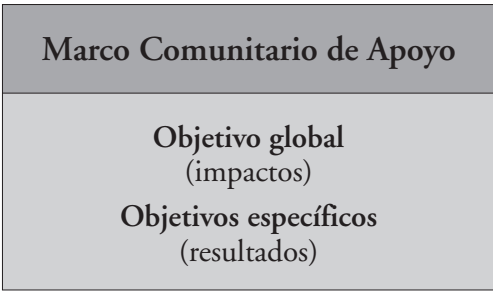

\begin{tabular}{|c|}
\hline $\begin{array}{c}\text { Documento único de } \\
\text { Programación }\end{array}$ \\
\hline $\begin{array}{c}\text { Objetivo global } \\
\text { (impactos) }\end{array}$ \\
$\begin{array}{c}\text { Objetivos específicos } \\
\text { (resultados) }\end{array}$ \\
\hline
\end{tabular}
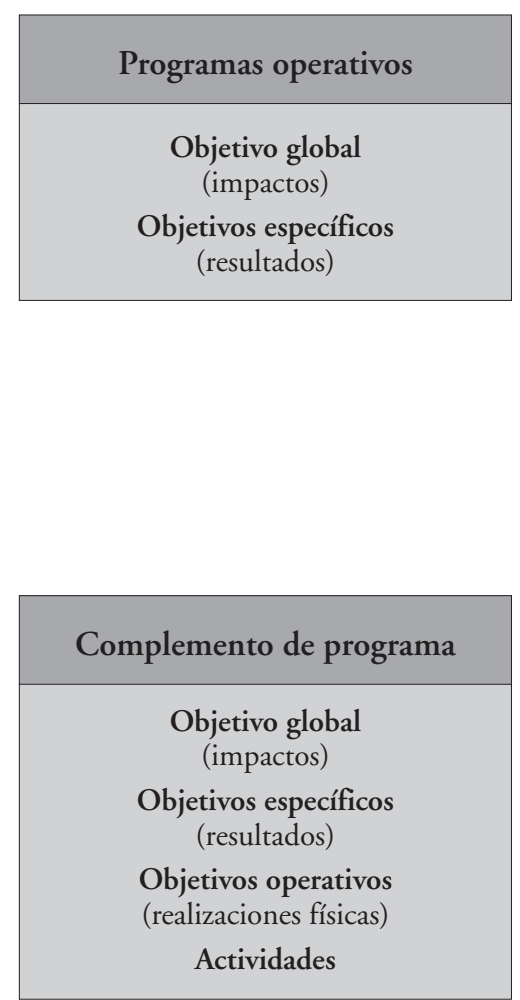

Complemento de programa

Objetivo global (impactos)

Objetivos específicos (resultados)

Objetivos operativos (realizaciones)

Actividades 


\section{El seguimiento y la evaluación de los programas comunitarios}

Pero ¿qué entiende la Comisión Europea por "seguimiento" y "evaluación"?

El Esquema 3 describe de forma gráfica la secuencia metodológica genérica que sería necesario desarrollar en todas y cada una de las acciones cofinanciadas para responder a las demandas de seguimiento y evaluación que ha solicitado la Comisión, en reiteradas ocasiones, a los Estados beneficiarios de los Fondos.

Para orientar y ayudar a los organismos coordinadores y ejecutores de Fondos Europeos, la Comisión Europea -directamente y a través de sus diferentes Direcciones Generales- ha elaborado y publicado diversos documentos que pueden servir de guía para la puesta en práctica de los sistemas de seguimiento y evalua- ción. Con el objetivo de acercar esas líneas directrices a las personas que tienen que llevarlas a cabo, en este apartado se sintetizan, recordando algunos conceptos y presentando algunos ejemplos.

\subsection{La distinción entre "seguimiento" y "evaluación"}

Una vez definidas y puesto en marcha las actuaciones concretas, es necesario y conveniente, como ya se apuntó en el apartado 1 (referido a la justificación de la necesidad de establecer un sistema de seguimiento y evaluación), realizar el seguimiento y -posteriormente- la evaluación de las mismas.

Como se indicó al comienzo del apartado 2.1. ("La lógica general de las intervenciones comunitarias"), la clasificación que utiliza la Comisión diferencia entre recursos utilizados, actividades realizadas (medidas, actuaciones...), realizaciones (referidas a los bienes y/o servicios producidos), resultados (referidos a los efectos directos e inmediatos) e impactos (referidos a los efectos a largo plazo). Recoger información de forma continua de estos cinco ámbitos resulta poco factible en la realidad. De hecho, lo que en el ámbito del seguimiento y la evaluación suele recomen-

\section{Esquema 3 \\ Filosofía genérica y objetivos de los métodos de seguimiento y evaluación vigentes para las acciones cofinanciadas}

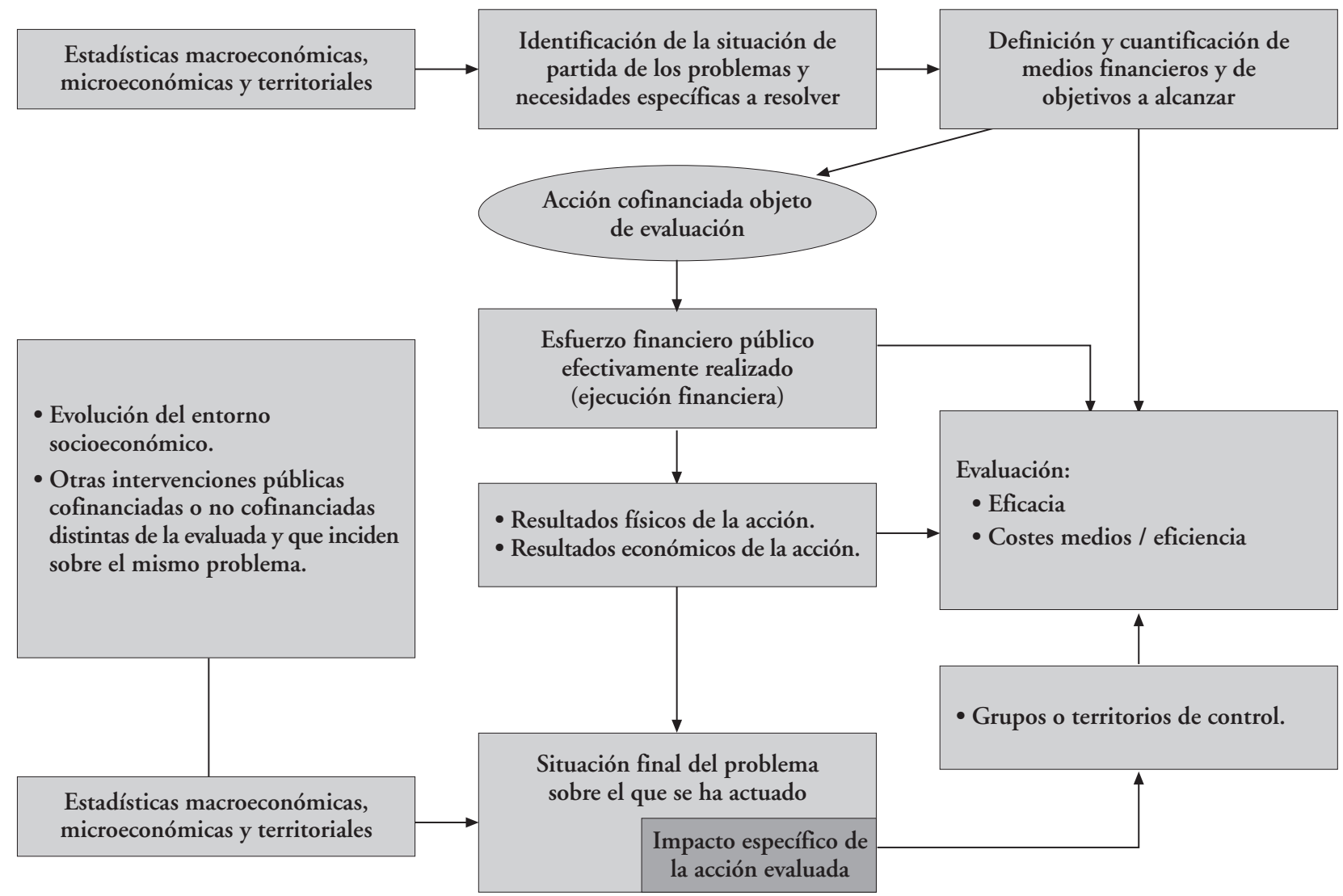


darse y considerarse adecuado es que los organismos gestores recojan información relativa a los recursos, a las actividades, a las realizaciones y -en algunos casos- a los resultados de sus acciones, y que la información sobre resultados e impactos se recopile en evaluaciones periódicas, a través de estudios específicos de casos o muestras de los colectivos atendidos. La experiencia ha demostrado que si existe un sistema de seguimiento bien diseñado y que se alimenta de forma continua, las evaluaciones -que son investigaciones esporádicas- llegan a conclusiones y recomendaciones más útiles para la programación y la gestión. De lo contrario, los evaluadores tendrán que dedicar gran parte de su tiempo a realizar un

\section{Recuadro 3 \\ El seguimiento y la evaluación}

El seguimiento es el examen periódico de la ejecución de un programa, para comprobar si la utilización de los recursos, los calendarios de trabajo, las realizaciones y los resultados esperados se están llevando a cabo de acuerdo con el plan trazado. Se basa en un sistema de información alimentado principalmente por los gestores, referido, sobre todo, a los objetivos operativos (realizaciones). Su objetivo principal es ayudar a los responsables de las actuaciones públicas (coordinadores y gestores) en la toma de decisiones relativas a planificación y ejecución.

La evaluación es una investigación objetiva y sistemática para emitir juicios de valor sobre una intervención pública, interpretando y analizando con métodos rigurosos la información incluida en el sistema de seguimiento y otra información recogida y/o producida especialmente. Su misión es determinar la pertinencia, la eficacia, la eficiencia, el impacto, la calidad y la sostenibilidad de todas las actividades, para ayudar a mejorar las actuaciones que se hallan aún en marcha y en la planificación y ejecución de programas futuros. trabajo de recopilación de información básica dispersa entre los organismos gestores, de difícil homogeneización y análisis.

Según el momento de la intervención en que se realice la evaluación, ésta tendrá unos objetivos diferentes y se referirá a unas cuestiones específicas, tal como se describe en los siguientes recuadros.

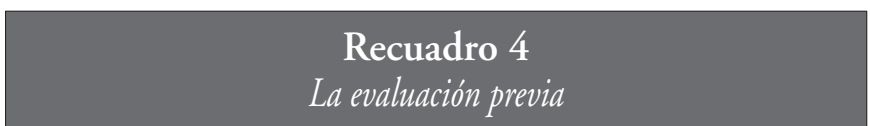

La evaluación previa (o "exante") se realiza antes de la implantación del programa. Valora si las necesidades están correctamente diagnosticadas, si las actividades, la estrategia y los objetivos propuestos son pertinentes, si son coherentes entre sí y con las orientaciones nacionales y comunitarias, si los impactos previstos son realistas, etc. Evidentemente, para que una evaluación previa pueda llevarse a cabo, la intervención (el programa) tiene que tener un mínimo de precisión.

Entre otros, debe analizar los siguientes elementos:

- la relación, pertinencia y coherencia entre los objetivos generales, los objetivos especificos y las medidas incluidas en el programa;

- la coherencia de la estrategia y los objetivos seleccionados con las necesidades y las características de la zona y el grupo objetivo a atender;

- la existencia y pertinencia de indicadores de recursos, realización, resultados e impactos en relación con cada uno de los niveles de la intervención;

- la fiabilidad de la valoración de los efectos potenciales y del nivel de cuantificación de los objetivos.

En el siguiente Esquema se resumen los componentes clave de la evaluación previa fijados en los artículos 40 y 41 del Reglamento por el que se establecen disposiciones generales sobre los Fondos Estructurales (CE 1260/99).

\section{Esquema 4 Componentes clave de la evaluación previa}

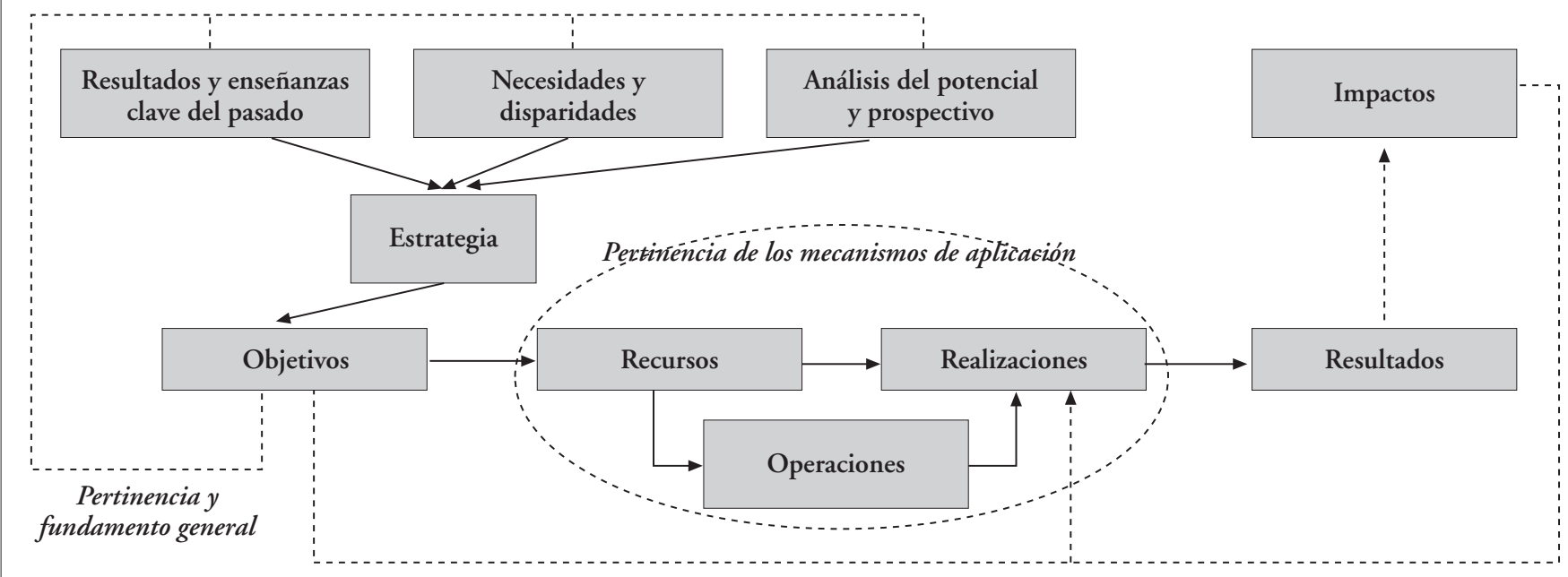

Eficacia

Fuente: Comisión Europea, 1999c:6. 


\section{Recuadro 5}

La evaluación intermedia

La evaluación intermedia se realiza durante el período de puesta en práctica del programa. Analiza los primeros resultados de las intervenciones, la pertinencia de los objetivos previstos y el grado de consecución de los mismos. Sus conclusiones deben incluir recomendaciones útiles para reorientar la intervención, en caso de que se considere necesario, a la vez que apoyar las programaciones futuras.

Se centra, sobre todo, en las siguiente cuestiones:

- Examen de las primeras realizaciones y resultados, determinando el grado de eficacia alcanzado hasta el momento.

- Estudio de la forma en que se han llevado a cabo las intervenciones previstas, y si se ha realizado 'de facto' alguna modificación a los objetivos iniciales, valorando la pertinencia y necesidad de estas modificaciones.

- Valoración de la calidad, utilidad y la pertinencia del sistema de seguimiento y los indicadores utilizados.

\section{Recuadro 6}

La evaluación posterior

La evaluación posterior (o final) se lleva a cabo una vez finalizada la intervención ${ }^{1}$. Recapitula y juzga el conjunto del programa, comparando los objetivos previstos con los realmente alcanzados. Sus conclusiones deben incluir recomendaciones y lecciones de la experiencia que permitan apoyar las programaciones futuras.

Es recomendable que, al menos, analice las siguiente cuestiones:

- La utilización de los recursos;

- la cobertura;

- los efectos positivos y negativos, tanto los previstos como los no previstos;

- La eficacia y la eficiencia;

- La utilidady la sostenibilidad de los resultados e impactos conseguidos.
El Esquema 5 refleja la interacción entre un programa y su evaluación en sus diferentes fases. Las flechas de línea discontinua indican que en la evaluación se obtiene y procesa información sobre los resultados e impactos de la intervención. Las flechas de línea continua indican el momento de la evaluación y, asimismo, que las conclusiones y recomendaciones de esa evaluación deben incidir en el programa y en su aplicación o en las medidas que le den continuidad. Las flechas superiores de trazo grueso indican que la evaluación debe aprovechar los resultados de evaluaciones anteriores, es decir, que la evaluación intermedia debe tener presentes los resultados de la evaluación previa, y la evaluación posterior las conclusiones de la evaluación intermedia.

La normativa comunitaria referida al seguimiento y la evaluación ha sufrido cambios importantes entre la vigente hasta 1999 y la vigente a partir del año 2000. Los principales cambios son: la mayor precisión (y reforzamiento) de las referencias sobre

$\checkmark$ la necesidad y obligatoriedad del seguimiento y de los tres tipos de evaluación;

$\checkmark$ la precisión de quién deberá asumir la responsabilidad del seguimiento y de cada una de las evaluaciones;

$\checkmark$ el aumento del grado de concreción de los objetivos del seguimiento y la evaluación;

$\checkmark$ el aumento del grado de implicación y de las responsabilidades de los Estados miembros; y

$\checkmark$ la vinculación de la calidad y los resultados de las evaluaciones con repercusiones financieras para los Estados (o regiones): la reserva de eficacia.

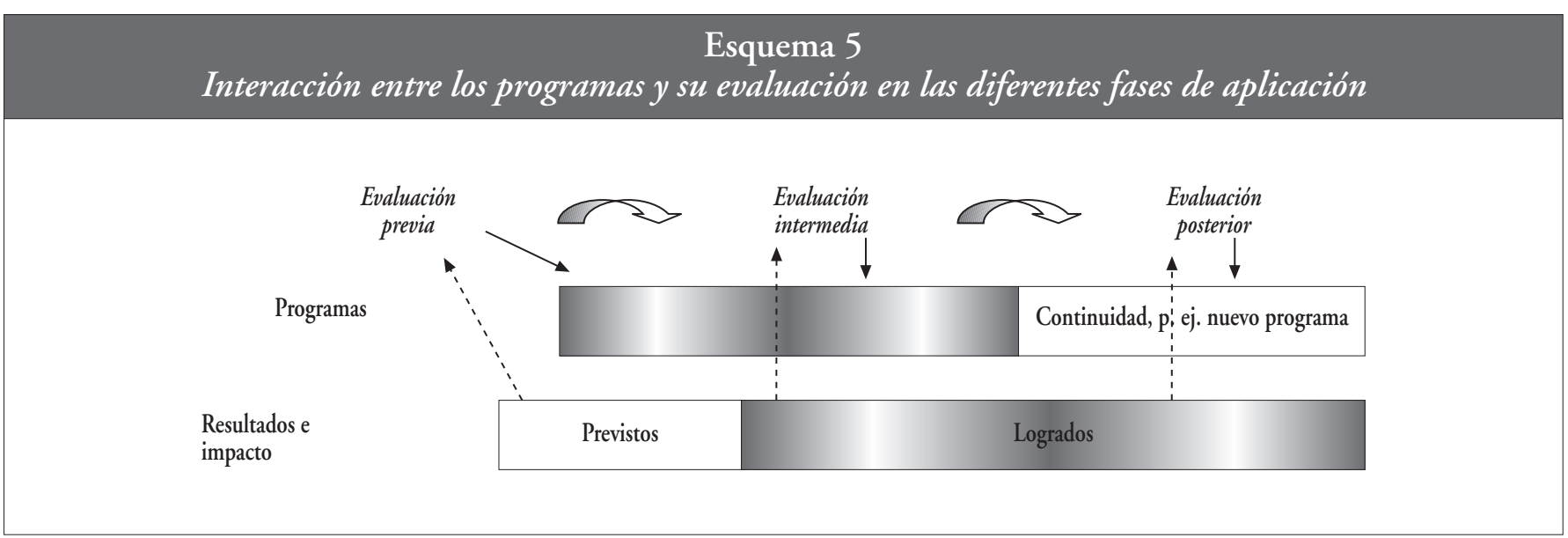


Un resumen comparativo de los sistemas de seguimiento y evaluación entre los dos períodos de programación se presenta a continuación:

\begin{tabular}{|c|c|}
\hline \multicolumn{2}{|c|}{$\begin{array}{l}\text { Cuadro } 4 \\
\text { e el seguimiento de las acciones estructurales } \\
\text { ídos } 1994-99 \text { y 2000-06 }\end{array}$} \\
\hline PERÍODO 1994-1999 & PERÍODO 2000-2006 \\
\hline \multicolumn{2}{|c|}{ ESTADO MIEMBRO } \\
\hline \multirow{2}{*}{$\begin{array}{l}\text { No hay ninguna disposición reglamentaria específica sobre las responsabilidades del } \\
\text { Estado miembro. El Estado miembro asume la responsabilidad general de la ejecución } \\
\text { y del seguimiento, así como de la eficacia de las intervenciones. }\end{array}$} & $\begin{array}{l}\text { El Estado miembro asume la responsabilidad general de la ejecución y del seguimiento, } \\
\text { así como de la eficacia de las intervenciones. }\end{array}$ \\
\hline & $\begin{array}{cl}\text { Responsabilidad de la autoridad de gestión: } \\
\checkmark & \text { Recopilación y transmisión de los datos financieros y estadísticos sobre la } \\
& \text { realización de las intervenciones. } \\
\checkmark & \text { Informe anual de ejecución. } \\
\checkmark & \text { Regularidad de las operaciones. } \\
\checkmark & \text { Respeto de las políticas comunitarias. } \\
\checkmark & \text { Información y publicidad sobre la intervención. } \\
\checkmark & \text { Organización de la evaluación intermedia. } \\
\checkmark & \text { Encuentro anual con la Comisión (examen de los resultados del año anterior). } \\
& \text { en su caso, adaptación de las modalidades de seguimiento o gestión a raíz de } \\
& \text { las recomendaciones de la Comisión. } \\
\checkmark & \text { Adaptación del complemento de programa, por iniciativa propia o a petición } \\
& \text { del Comité de seguimiento. }\end{array}$ \\
\hline $\begin{array}{l}\text { Responsabilidad del Comité de seguimiento: } \\
\text { No hay ninguna definición precisa del papel del Comité de seguimiento. }\end{array}$ & $\begin{array}{cl}\text { Responsabilidad del Comité de seguimiento: } \\
\checkmark & \text { Aprobación del complemento del programa; } \\
\checkmark & \text { Aprobación de las propuestas de modificación de la intervención; } \\
\checkmark & \text { Aprobación de los criterios de selección de las operaciones financiadas; } \\
\checkmark & \text { Seguimiento de las realizaciones y evaluación de los progresos realizados para } \\
& \text { lograr los objetivos de las intervenciones; } \\
\checkmark & \text { Aprobación del informe anual de ejecución. }\end{array}$ \\
\hline & $\begin{array}{l}\text { Indicadores de seguimiento e informe anual de ejecución: } \\
\text { Definición reglamentaria de su objetivo y contenido. }\end{array}$ \\
\hline \multicolumn{2}{|c|}{ COMISIÓN } \\
\hline & $\begin{array}{l}\text { Relaciones con la autoridad de gestión: } \\
\qquad \begin{array}{ll}\checkmark & \text { Encuentro anual con la autoridad de gestión; } \\
\checkmark & \text { En su caso, observaciones o recomendaciones de adaptación dirigidas al Estado } \\
& \text { miembro o a la autoridad de gestión. }\end{array}\end{array}$ \\
\hline 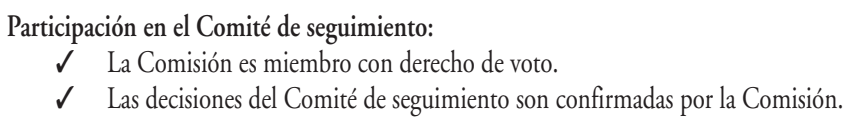 & $\begin{array}{l}\text { Participación en el Comité de seguimiento: } \\
\quad \checkmark \quad \text { La Comisión es miembro con carácter consultivo. }\end{array}$ \\
\hline $\begin{array}{l}\text { Informe anual: } \\
\quad \checkmark \quad \text { Examen del informe anual. }\end{array}$ & $\begin{array}{l}\text { Indicadores de seguimiento e informe anual de ejecución: } \\
\checkmark \text { Aprobación del informe anual. } \\
\checkmark \text { Propuestas metodológicas para los indicadores de seguimiento. }\end{array}$ \\
\hline \multicolumn{2}{|c|}{ REFERENCIAS } \\
\hline Reglamento de coordinación: artículo 25. & Reglamento general: artículos 34, 35, 36, 37 y 46 . \\
\hline
\end{tabular}

Fuente: Comisión Europea, 2000:28. 


\section{Cuadro 5 \\ Comentarios comparativos sobre la evaluación de las acciones estructurales entre los períodos 1994-99 y 2000-06}

PERÍODO 1994-1999

PERÍODO 2000-2006

\section{EVALUACIÓN PREVIA}

$\checkmark \quad$ La Comisión y el Estado miembro deben realizar, en colaboración, una evaluación previa que incluya una referencia a la situación medioambiental.

$\checkmark$ La Comisión apreciará los planes teniendo en cuenta la evaluación previa. $\checkmark$ La responsabilidad principal de la evaluación previa incumbe al Estado miembro.

$\checkmark$ El objeto de la evaluación previa está definido. Debe prestarse una atención especial a la evaluación de la situación medioambiental y del mercado laboral, así como a la igualdad de oportunidades entre hombres y mujeres.

$\checkmark$ La evaluación previa está integrada en los planes.

\section{EVALUACIÓN INTERMEDIA}

$\checkmark \quad$ La evaluación intermedia no está prevista en ninguna disposición reglamentaria. En la práctica, por iniciativa de la Comisión y en cooperación con ella, los Estados miembros han organizado evaluaciones intermedias, con ayuda de evaluadores independientes, siguiendo modalidades precisadas en los MCA.

$\checkmark \quad$ No hay ninguna disposición reglamentaria que defina las obligaciones de recopilación y presentación de datos.

$\checkmark$ No hay ninguna disposición reglamentaria que defina las funciones de evaluación del Comité de seguimiento. $\checkmark \quad$ La evaluación intermedia incumbe al Estado miembro en cooperación con la Comisión, la que aprecia su pertinencia.

$\checkmark$ El objeto de la evaluación intermedia está definido.

$\checkmark \quad$ La evaluación intermedia es organizada por la autoridad de gestión de la intervención y realizada por un evaluador independiente. Debe estar terminada antes del 31 de diciembre de 2003.

$\checkmark \quad$ De cara a las distintas evaluaciones, la autoridad de gestión debe establecer un mecanismo de recopilación de datos financieros y estadísticos para las evaluaciones intermedias y posteriores, y proporcionar la información necesaria para la evaluación posterior.

$\checkmark$ Los resultados de la evaluación intermedia son examinados por el Comité de seguimiento, que puede proponer adaptaciones de la intervención en función de ellos.

$\checkmark \quad$ La evaluación intermedia debe actualizarse antes de finales de 2005 con vistas a preparar las intervenciones posteriores.

\section{EVALUACIÓN POSTERIOR}

$\checkmark \quad$ La Comisión y el Estado miembro deben realizar en colaboración una evaluación posterior con el fin de apreciar el impacto de las acciones en relación con los objetivos fijados. $\checkmark \quad$ La Comisión tiene la responsabilidad principal de la evaluación posterior, en colaboración con el Estado miembro.

$\checkmark$ El objeto de la evaluación posterior está definido.

$\checkmark \quad$ La evaluación posterior es realizada por un evaluador independiente, a más tardar tres años después de finalizado el período de programación.

\section{RESERVA DE EFICACIA}

\begin{tabular}{|c|c|}
\hline & $\begin{array}{l}\checkmark \text { Antes del } 31 \text { de abril de 2004, la Comisión, basándose en la propuesta del Estado } \\
\text { miembro, asignará a los P.O. o DOCUP considerados eficaces los créditos nacionales } \\
\text { reservados al principio del período en concepto de "reserva de eficacia" (4\%). } \\
\checkmark \quad \text { La eficacia se juzgará mediante indicadores, definidos por el Estado miembro en } \\
\text { concertación con la Comisión, que reflejen la eficacia, la gestión y la ejecución } \\
\text { financiera. }\end{array}$ \\
\hline \multicolumn{2}{|c|}{ REFERENCIA } \\
\hline $\begin{array}{l}\text { Reglamento marco: artículo } 6 . \\
\text { Reglamento de coordinación: artículo } 26 .\end{array}$ & Reglamento general: artículos 40, 41, 42, 43 y 44 . \\
\hline
\end{tabular}


De todo lo anterior se puede concluir que existen una serie de cuestiones clave a ser considerados en una evaluación: pertinencia, utilidad, perdurabilidad (sostenibilidad), eficacia y efi- ciencia. La relación entre estos elementos y la lógica de la intervención de un programa se puede resumir de la siguiente forma:

\section{Esquema 6}

\section{Cuestiones clave de la evaluación}

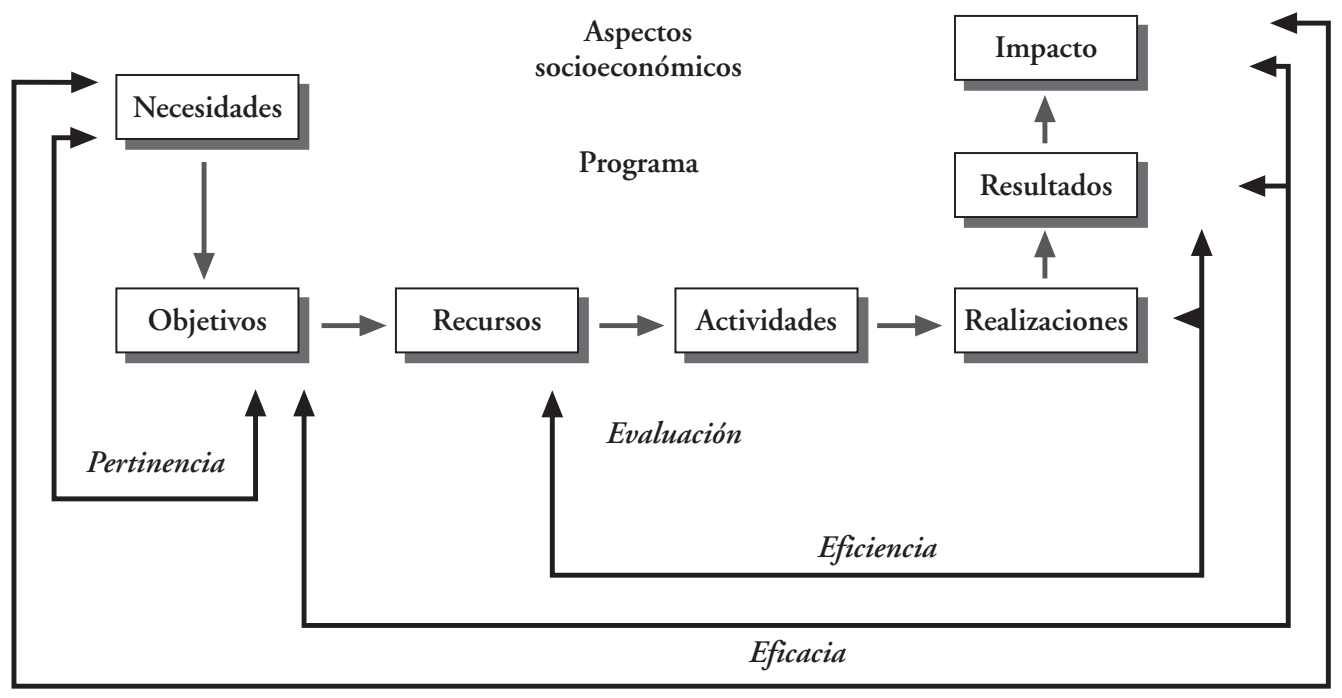

Utilidad y perdurabilidad

Fuente: Comisión Europea, 1999b:11.

Si se revisan con cierta atención las definiciones que se han presentado hasta el momento, puede apreciarse fácilmente que la posibilidad de alcanzar los objetivos del seguimiento y la evaluación se encuentra relacionada, de forma muy estrecha, con dos cuestiones estratégicas desde el punto de vista metodológico: por un lado, con la calidad de la programación; y, por otro, con la existencia diferenciada de un sistema (administrativo) de seguimiento y otro de evaluación. Por ello, el éxito o el fracaso de cualquier metodología de evaluación depende de manera crítica de la calidad de la programación (su "evaluabilidad" de acuerdo al término acuñado a finales de 1996 cuando se lanzó un programa de análisis de la programaciones operativas españolas cofinanciadas) y, sobre todo, de la cantidad y calidad de la información que pueda generar cada organismo gestor durante la tramitación de los proyectos y expedientes.

\subsection{Herramientas para el seguimiento y la evaluación}

Bien, pero... ¿qué pasos generales hay que seguir para realizar un seguimiento y una evaluación satisfactorios? A continuación se describen las herramientas utilizadas para el seguimiento y la evaluación, y los pasos generales a seguir para realizar un seguimiento y una evaluación satisfactorios.

\section{a) Los indicadores}

Los indicadores son medidas específicas y objetivamente verificables de los cambios o los efectos de una actividad. Proporcionan una escala con la que puede medirse un cambio. Los indicadores sirven de patrón de medida para mostrar: (a) el uso de los recursos y el progresos de una actividad (indicadores de recursos y de actividad); (b) la obtención de productos y servicios (realizaciones), resultados e impactos (indicadores de realización, de resultados y de impactos); y (c) la influencia de factores externos (indicadores del contexto). Se utilizan para cuantificar y simplificar la información sobre fenómenos complejos.

Veamos algunos ejemplos de indicadores relativos al proyecto de infraestructura que ya hemos utilizado en el Cuadro 1. 


\begin{tabular}{|c|c|c|}
\hline \multicolumn{3}{|c|}{$\begin{array}{l}\text { Cuadro } 6 \\
\text { s a un proyecto de infraestructura }\end{array}$} \\
\hline & DESCRIPCIÓN & INDICADORES \\
\hline Realización & • Una nueva autopista. & $\begin{array}{l}\text { - De ejecución financiera: coste por km. } \\
\text { - De ejecución física: número de km. construidos. }\end{array}$ \\
\hline Resultados & $\begin{array}{l}\text { - Reducir el tiempo de recorrido. } \\
\text { - Reducir los costes de transporte. } \\
\text { - Mejorar la seguridad. }\end{array}$ & $\begin{array}{l}\text { - \% tiempo de recorrido nuevo/ tiempo anterior. } \\
\text { - Ahorro de tiempo (en minutos). } \\
\text { - \% costes de transporte nuevo/ coste anterior. } \\
\text { - Reducción de costes }(\%) \text {. } \\
\text { - Accidentes actuales por automóvil-km. } \\
\text { - \% accidentes actuales/ anteriores. }\end{array}$ \\
\hline Impactos & $\begin{array}{l}\text { - Incrementar los flujos interregionales de personas y } \\
\text { mercancías. } \\
\text { - Incrementar las actividades socioeconómicas. }\end{array}$ & $\begin{array}{l}\text { - \% de incremento de toneladas transportadas. } \\
\text { - \% de incremento de automóviles en circulación. } \\
\text { - \% de incremento de personas desplazadas. } \\
\text { - Variación del PIB regional por persona y por empleado. } \\
\text { - Variación del empleo neto en la región. }\end{array}$ \\
\hline
\end{tabular}

Fuente: Elaboración propia sobre la base de: Comisión Europea, 1999b:10.

Como puede verse en el ejemplo anterior, en función del esquema lógico de programación utilizado, los indicadores pueden clasificarse de la siguiente forma:

- En primer lugar, los indicadores básicos del programa. Se trata de:

- Los indicadores de realización, que sirven para medir:

- La ejecución financiera y/o los medios utilizados (en general relativos a los gastos efectuados con ocasión de la realización de una acción: coste en euros de una autopista por km., coste de un programa de formación por alumno, coste de un centro de servicios tecnológico por metro cuadrado, etc.).

- El avance o ejecución física de una acción (en general indicadores físicos: número de km., número de personas, porcentaje de empresas que han obtenido una ayuda, etc.).

- Los indicadores de resultados: para medir los resultados, los efectos directos o inmediatos, la calidad o el rendimiento que la realización de la acción consigue. Estos indicadores pueden ser físicos (porcentaje de reducción del tiempo de recorrido, porcentaje de éxito de los alumnos, número de accidentes en una autopista, porcentaje de llamadas telefónicas fallidas por línea y año, etc.) o financieros (inversiones inducidas, disminución de los costes de transporte, etc.).
- Los indicadores de impactos: para medir el impacto que las acciones realizadas y los resultados obtenidos ha permitido lograr. Es decir, miden si los objetivos específicos alcanzados han ayudado a obtener los objetivos globales establecidos.

- A partir de estos indicadores del programa es posible calcular otros indicadores complementarios:

- Los indicadores de eficacia: los que permiten medir lo que se ha conseguido en relación a lo que se pretendía, es decir, medir los resultados o los impactos obtenidos en relación con los resultados o impactos previstos o estimados.

- Los indicadores de eficiencia: los que permiten medir las realizaciones, los resultados y los impactos obtenidos en relación con los recursos movilizados, sobre todo financieros.

Estos indicadores de eficacia y de eficiencia se aplican a todas las etapas de una acción, es decir, tanto a las realizaciones como a los resultados y a los impactos. Permiten comparar lo que se ha obtenido en relación con lo que se había previsto (eficacia) o en relación con los medios movilizados (eficiencia).

Las dos clasificaciones son complementarias, no excluyentes. El siguiente cuadro pone de manifiesto la complementariedad entre indicadores básicos del programa y los indicadores de eficacia y de eficiencia: 


\begin{tabular}{|l|l|l|l|}
\hline \multicolumn{2}{|c|}{$\begin{array}{c}\text { Cuadro } 7 \\
\text { Complementariedad entre indicadores básicos del programa e indicadores de eficacia y de eficiencia }\end{array}$} \\
\hline \multicolumn{1}{|c|}{$\begin{array}{c}\text { INDICADORES BÁSICOS } \\
\text { DEL PROGRAMA }\end{array}$} & $\begin{array}{c}\text { INDICADORES DE } \\
\text { EFICACIA }\end{array}$ & $\begin{array}{c}\text { INDICADORES DE COSTES } \\
\text { MEDIOS (EFICIENCIA) }\end{array}$ \\
\hline Realización & $\begin{array}{c}\text { Indicadores de ejecución financiera. } \\
\text { - Indicadores de realización física. }\end{array}$ & $\begin{array}{l}\text { - Ejecución realizada/prevista. } \\
\text { Realización efectuada/prevista. }\end{array}$ & $\begin{array}{l}\text { - Realización efectuada/costes. } \\
\text { De ejecución física: número de km. } \\
\text { construidos. }\end{array}$ \\
\hline Resultados & Indicadores de resultados. & Resultados obtenidos/previstos. & Resultados obtenidos/costes. \\
\hline Impactos & Indicadores de impactos. & Impacto obtenido/previsto. & Impacto obtenido/costes. \\
\hline
\end{tabular}

Fuente: Elaboración propia sobre la base de: Comisión Europea, 1999b:12.

En relación a lo anterior, hay que señalar algunas dificultades que pueden entrañar la utilización de los indicadores, ya que su disponibilidad y utilización no resultan fáciles. Hay que recordar, además, que información no es lo mismo que conocimiento (PATTON, 2001: 329).

- Las dificultades para comprobar las relaciones de causalidad entre las acciones efectuadas, los resultados obtenidos y el impacto apreciado. Una mejora de la situación económica puede deberse, por ejemplo, a factores exógenos a los derivados de la intervención cofinanciada. En relación a esto, puede resultar interesante utilizar, por ejemplo, métodos que permitan comparar el impacto de una acción sobre un grupo, con un grupo similar que no se haya beneficiado de esa acción (lo que se llama un grupo de control). Las evaluaciones intermedias y posteriores se utilizan para este tipo de verificación, entre otras cosas.

- La relativa complejidad de los métodos de medida. Las realizaciones y los resultados son relativamente fáciles de medir, puesto que se sitúan muy cerca de la propia acción. En cambio, para medir el impacto es preciso dejar pasar cierto tiempo para que el impacto se manifieste, y utilizar métodos de medición y de investigación relativamente complejos. Además, el impacto es, a menudo, el resultado de varias acciones, y es preciso, por lo tanto, utilizar instrumentos que permitan analizar el efecto acumulado de varios tipos de acciones.

- La no disponibilidad dentro de los plazos de las informaciones necesarias para calcular los indicadores.

- La dificultad de sumar ciertos indicadores. Los indicadores financieros pueden agregarse, pero los indicadores físicos son mucho más delicados de manejar cuando se desea hacer agregaciones. Su naturaleza es, por lo general, demasiado distinta como para permitir una mera suma aritmética.

Otro elemento a tener en cuenta es el relativo al contexto, ya que los cambios de tipo económico, social, político, jurídico, logístico, meteorológico, etc., pueden condicionar de forma significativa la ejecución y/o resultados de las acciones. Por ello, también será necesario establecer para el seguimiento una serie de indicadores del contexto que se consideren relevantes para cada acción. Estos indicadores ayudan a entender las condiciones bajo las cuales se ha desarrollado un programa (PATTON, 2002: 231; WEISS, 2002: 229).

\section{b) Situación de partida e indicadores de contexto}

\begin{tabular}{|c|}
\hline $\begin{array}{c}\text { Recuadro } 7 \\
\text { La necesidad de definir la situación de partida }\end{array}$ \\
\hline $\begin{array}{l}\text { "Los planes presentados en virtud de los objetivos números } 1,2 \text { y } 3 \text { (...) } \\
\text { incluirán: a) una descripción, a ser posible cuantificada, de la situación } \\
\text { actual, bien en materia de disparidades, atrasos y posibilidades de desarrollo } \\
\text { en el caso de las regiones del objetivo } n^{\circ} 1 \text {, bien en materia de reconversión } \\
\text { en las regiones del objetivo } n^{\circ} 2 \text {, o bien en materia de desarrollo de los } \\
\text { recursos humanos y de politica de empleo en el Estado miembro y en las } \\
\text { regiones del objetivo no } 3 \text { (....)". }\end{array}$ \\
\hline
\end{tabular}

Fuente: Artículo 16 del Reglamento por el que se establecen disposiciones generales sobre los Fondos Estructurales (CE 1260/99).

Como se reproduce en el recuadro anterior, el artículo 16 del Reglamento de los Fondos Estructurales establece que los planes de desarrollo deben incluir una descripción cuantificada de las disparidades, atrasos y posibilidades de desarrollo de las regiones en cuestión. Los indicadores de contexto se utilizan para cumplir 
con dicha disposición y forman parte del proceso de programación. Al mismo tiempo, ofrecen una base para:

- los análisis socioeconómicos y estratégicos, que constituyen la base de la estrategia del programa;

- el seguimiento del contexto general;

- la ejecución y definición de objetivos cuantificados;

- la evaluación de los impactos socioeconómicos del programa.

La situación de partida (o de base) hace referencia al valor inicial respecto del cual se comparan posteriormente los indicadores de contexto y los de impacto. Dichos datos deben establecerse en relación con los objetivos del programa y pueden incluir, por ejemplo, el número inicial de empleos industriales en la región o el volumen de inversión privada en un sector o industria en un momento determinado. En la práctica, existen grandes lagunas en la disponibilidad de datos sobre una serie de aspectos clave, especialmente en lo que se refiere a las pequeñas y medianas empresas (PYMEs).

Los elementos de la situación de base también son indispensables para que los indicadores del programa sean significativos. Así por ejemplo, si el objetivo de una medida especifica es aumentar el número de PYMEs en la región, el dato de partida más importante será el número de PYMEs existentes al comienzo del programa. Una vez obtenida esta información, podrá deducirse más concretamente que, por ejemplo, el 20\% de las empresas existentes en una región subvencionable se beneficia de la asistencia de los Fondos Estructurales.

El contenido de esta información puede volver a definirse durante la ejecución del programa. Los datos de la situación de partida pueden establecerse de forma que abarquen adecuadamente todos los objetivos incluidos en la programación. En algunos casos, podría ser conveniente recoger datos específicos sobre los beneficiarios del programa, como por ejemplo las PYMEs, o los desempleados de larga duración. Por otra parte, otra serie de indicadores más detallados por sector de actividad, tamaño o sexo facilitarán una descripción más completa de los beneficiarios y permitirán establecer comparaciones con intervenciones anteriores y con iniciativas de otras regiones.

En general, deben cuantificarse los objetivos correspondientes a los programas y las acciones. De lo contrario, será más difícil evaluar en qué medida se están cumpliendo los objetivos originales. Los datos necesarios para cuantificar los objetivos del programa suelen estar disponibles. La cuantificación puede utilizar los datos de la situación de partida y los valores de referencia y de comparación de anteriores ejercicios de seguimiento y evaluación, tales como el coste medio de un empleo creado o conservado en un sector dado.

El nivel de cuantificación requerido depende del tipo de intervención. En el caso de las medidas de infraestructuras, es más sencillo establecer objetivos cuantificados al comienzo (por ejemplo, número de kilómetros de carretera que serán construidos), basándose en las características técnicas y económicas de los proyectos que se van a financiar. No obstante, con frecuencia no es posible medir de forma precisa el objetivo perseguido, puesto que en algunos casos el número de beneficiarios (PYMEs o trabajadores formados) no puede establecerse de antemano. En tales casos, que no se prestan a la cuantificación directa, es más adecuado establecer una serie de objetivos posibles, cuyos valores pueden refinarse durante la fase de ejecución.

Fuentes de información. Los datos de la situación de base se obtienen, en primer lugar, de las estadísticas oficiales. No obstante, en algunos casos, estas fuentes pueden presentar algunas deficiencias, entre las que hay que destacar las siguientes:

- falta de datos a una escala geográfica apropiada;

- retrasos en la publicación de datos (por ejemplo, los datos de Eurostat sobre el PIB per capita se publican con un retraso de dos a tres años);

- ausencia de datos en relación con los requisitos del programa (por ejemplo, la distinción entre trabajadores a tiempo completo y a tiempo parcial podría no figurar en las estadísticas oficiales);

- ausencia de datos suficientemente desglosados por sector.

En algunos casos, las estadísticas oficiales tendrán que completarse con encuestas, estudios específicos o, posiblemente, indicadores indirectos (por ejemplo, las cifras de negocios de las PYMEs pueden facilitar información acerca de su grado de competitividad).

Por otra parte, desde 1992-1993 la Comisión elabora, en cooperación con los Estados miembros, un sistema común de indicadores cuantificados para medir las diferencias de desarrollo. Se elaboró una lista inicial de indicadores de contexto, divididos en doce grupos: indicadores básicos (PIB per capita, etc.), transporte por carretera, transporte por ferrocarril, telecomunicaciones, energía, recursos hidrológicos, medio ambiente, educación y formación, investigación y desarrollo tecnológico, industria y servicios, agricultura y turismo. Estos datos cuantificados están incluidos en una base de datos, denominada "QUID". 


\section{c) Indicadores de seguimiento}

\begin{tabular}{|c|}
\hline $\begin{array}{l}\text { Recuadro } 8 \\
\text { Indicadores de seguimiento }\end{array}$ \\
\hline $\begin{array}{l}\text { "La autoridad de gestión y el Comité de seguimiento realizarán el } \\
\text { seguimiento por medio de indicadores fisicos y financieros definidos en el } \\
\text { programa operativo, el documento único de programación o el complemento } \\
\text { de programación. Al elaborar estos indicadores, deberán tenerse en cuenta } \\
\text { las indicaciones metodológicas y las listas de ejemplos de indicadores } \\
\text { publicados por la Comisión, asi como la clasificación de ámbitos de } \\
\text { intervención que proponga la Comisión (...). Los indicadores harán } \\
\text { referencia al carácter especifico de la intervención en cuestión, a sus } \\
\text { objetivos, así como a la situación socioeconómica, estructural y } \\
\text { medioambiental del Estado miembro, y de sus regiones, según proceda } \\
\text { (...). Entre estos indicadores figurarán, en particular, los elegidos para la } \\
\text { asignación de la reserva [de eficacia] (...).". }\end{array}$ \\
\hline $\begin{array}{l}\text { "Estos indicadores señalarán, respecto de las intervenciones en cuestión: } \\
\text { a) los objetivos específicos (...) de las medidas y los ejes prioritarios, y su } \\
\text { coherencia; b) el estado en que se encuentra la intervención en lo relativo } \\
\text { a realizaciones fisicas, resultados y, tan pronto como sea posible, su impacto } \\
(\ldots) \text {; c) el estado en que se encuentre el desarrollo del plan de financiación". }\end{array}$ \\
\hline $\begin{array}{l}\text { "Cuando la naturaleza de la intervención lo permita, las estadisticas } \\
\text { indicarán el sexo de las personas atendidas y el tamaño de las empresas } \\
\text { beneficiarias". }\end{array}$ \\
\hline
\end{tabular}

Fuente: Artículo 36 del Reglamento por el que se establecen disposiciones generales sobre los Fondos Estructurales (CE 1260/99).

Según indica el Reglamento que rige los Fondos Estructurales, los MCA, los DOCUP y los P.O. deben incluir una información mínima que garantice el seguimiento adecuado de su aplicación. En la medida de lo posible, deben establecerse y cuantificarse los objetivos generales y específicos, así como los resultados previstos. El complemento del programa elaborado por el Estado miembro debe incluir una descripción pormenorizada de las medidas y una cuantificación de los objetivos operativos asociados.

Como ya se apuntó al principio de este apartado, el seguimiento es el examen periódico de los recursos, las realizaciones y los resultados de las intervenciones públicas. Los organismos coordinadores y ejecutores deberán definir (con ayuda de expertos externos si es necesario) los indicadores de recursos, realizaciones y resultados que integrarán el sistema de seguimiento. Serán los organismos gestores los que posteriormente recogerán la información necesaria para cumplimentar el sistema de seguimiento.

La teoría sobre indicadores enumera las características que los indicadores deben cumplir: (a) validez: que exista relación causal entre el indicador y lo que se quiere medir (la variable); (b) fiabilidad: que sean verificables y objetivos, es decir, que las diferencias entre las medidas obtenidas no se deban a la casualidad o al azar, que las medidas sean las mismas en mediciones repetidas, aunque sean realizadas por diferentes observadores; (c) sensibilidad: que el indicador y su unidad de medida reflejen los cambios, es decir, que la cantidad medida varíe cuando aparezca un cambio en la variable medida; $y(d)$ accesibilidad: que sea posible un cálculo lo suficientemente rápido a un coste aceptable, es decir, es recomendable no seleccionar un indicador si para recoger la información se necesita una cantidad de tiempo y recursos que se estima excesiva.

De hecho, la experiencia ha demostrado que la fase de definición y selección de indicadores de seguimiento es muy importante para garantizar la posterior factibilidad del uso del Sistema de seguimiento. Pero los indicadores de seguimiento seleccionados no sólo deben tener las características apuntadas en el párrafo anterior, sino también las siguientes:

$>$ relacionarse de forma directa con las actividades concretas que llevan a cabo los organismos gestores;

$>$ adecuarse al tipo de información que los organismos gestores pueden recoger o elaborar en su actividad diaria;

$>$ definirse de forma clara, es decir, no ser ambiguos; $y$

$>$ no ser muy numerosos.

Se definirán también los indicadores de impacto, que serán utilizados, entre otros, en las evaluaciones intermedias y posteriores, y en los estudios específicos que se considere oportuno llevar a cabo.

Un seguimiento eficaz exige la elaboración de indicadores de realizaciones en relación con todas o la mayor parte de las medidas. No obstante, en lo que se refiere a los indicadores de resultados y de impacto puede admitirse un enfoque algo más selectivo. Aunque estos últimos no puedan obtenerse de forma sistemática o a intervalos regulares, deben recogerse durante el proceso de evaluación.

Se definirán, además, los indicadores específicos del programa que se utilizarán para asignar a mitad del recorrido la reserva de eficacia general, tal como se establece en el Reglamento.

Las tareas preparatorias para establecer el sistema de seguimiento también servirán para detectar las deficiencias del sistema de información. Podrá ser necesario recurrir a asistencia técnica o a expertos independientes para solventar las posibles deficiencias y carencias, mejorar las condiciones generales de aplicación y aumentar la eficacia del seguimiento.

El organismo responsable del seguimiento, en su caso la autoridad de gestión, será competente para definir, sobre la base de las prioridades y de la capacidad, la estructura del sistema de seguimiento y el grado de detalle necesario para poder satisfacer las necesidades de los diferentes grupos usuarios (incluida la Comisión).

Será necesario garantizar el seguimiento regular de los progresos físicos y financieros de las medidas y, cuando sea posible, de los resultados. Aunque la disponibilidad de recursos de gestión y administrativos es un factor importante, sin embargo, como 
mínimo debe hacerse un seguimiento de los resultados de las medidas más relevantes del programa.

Es decir, el seguimiento deberá incluir:

- el seguimiento concreto de las realizaciones y de los resultados de las acciones,

- los progresos en la gestión y

- el seguimiento del contexto.

Este seguimiento puede realizarse: (a) de forma cuantitativa, con ayuda de indicadores; $y / 0$ (b) de forma descriptiva o cualitativa, a partir de distintos informes disponibles o de resultados de estudios o análisis.

En la medida de lo posible, será siempre preferible utilizar medidas cuantitativas, es decir, presentar las realizaciones efectuadas, los resultados obtenidos y el impacto alcanzado de forma cuantitativa, con ayuda de indicadores.

La definición de estos indicadores deberá permitir identificar mejor los objetivos que hayan de alcanzarse. Aun cuando resulte difícil su cuantificación, el esfuerzo de reflexión para definirlos puede ya resultar útil y clarificador.

Hasta el momento, el seguimiento de la ejecución financiera se puede considerar generalmente satisfactorio, pero el de las realizaciones físicas, los resultados y los impactos puede mejorarse.

\subsection{Aplicación de los indicadores de seguimiento}

A continuación se describen los principales aspectos del proceso de seguimiento.

\section{a. Recogida de datos}

Como ya se apuntó, la obtención de datos para el sistema de seguimiento es competencia de las autoridades responsables de la ejecución de la intervención. Por ello, debe fomentarse la utilización óptima de los sistemas de información operativos existentes, así como evitar la duplicación de tareas por distintos organismos. La información facilitada por las autoridades nacionales y regionales y la obtenida de los servicios estadísticos debe utilizarse exhaustivamente. Asimismo, deben dedicarse esfuerzos a consolidar o mejorar los datos existentes.

Como indica el Reglamento, tanto los datos financieros como los físicos deben recogerse, en la medida de lo posible, con arreglo a la nomenclatura sectorial propuesta por la Comisión.

\section{b. Análisis inicial de los datos}

Una vez recogidos, los datos deben ser elaborados y procesados, efectuándose una interpretación periódica de los mismos. Esto servirá de base al organismo responsable de la coordinación y del seguimiento para apoyar la toma de decisiones.

En general, este análisis forma parte de las tareas de las autoridades y organismos responsables de la intervención. Sin embargo, podrá recurrirse a expertos externos o a las organizaciones responsables de la recogida inicial de los datos cuando estos análisis resulten demasiado complejos.

\section{c. Presentación de los datos al Comité de seguimiento}

Recuadro 9
Comité de seguimiento
"Cada marco comunitario de apoyo o documento único de programación
y cada programa operativo será supervisado por un Comité de seguimiento."
"Los Comités de seguimiento serán creados por el Estado miembro (...).
Un representante de la Comisión y, cuando proceda, del BEI participará
con carácter consultivo en los trabajos del Comité. (...) estará presidido,
en principio, por un representante del Estado miembro o de la autoridad
de gestión."
"(...) El Comité de seguimiento comprobará la eficacia y el correcto
desarrollo de la intervención.(..) c) revisará periódicamente los avances
realizados en relación con el logro de los objetivos especificos de la
intervención; d) estudiará los resultados de la aplicación, en particular
la realización de los objetivos fijados para las distintas medidas (...); e)
estudiará y aprobará el informe anual y el informe final de ejecución
(...)”.

Fuente: Artículo 35 del Reglamento por el que se establecen disposiciones generales sobre los Fondos Estructurales (CE 1260/99).

Los comités de seguimiento son responsables de garantizar que las intervenciones tengan una aplicación eficaz y sean de calidad satisfactoria. Sus tareas incluyen el análisis de los progresos y, en particular, del grado de consecución de los objetivos cuantificados asociados a cada una de las medidas.

La información presentada a los comités de seguimiento debe incluir los siguientes elementos:

- datos de los sistemas de seguimiento (datos de situación de partida e indicadores de seguimiento);

- datos de la evaluación intermedia (incluida, en su caso, la revisión de los indicadores); $y$,

- datos provisionales sobre el contexto socioeconómico general y la adicionalidad del programa.

Los comités de seguimiento podrán decidir, sobre la base de las características de la intervención en cuestión, qué datos son necesarios y cuándo deberán estar disponibles. 
Para llevar a cabo el seguimiento de un MCA, un P.O. o un DOCUP, los comités de seguimiento deben recibir, con carácter anual, datos del seguimiento que abarquen la ejecución financiera, las realizaciones físicas y la gestión del programa.

Una vez que los sistemas de seguimiento sean operativos, se podrán medir, mediante los indicadores apropiados, tanto los resultados como la eficacia y elaborar estimaciones iniciales de los posibles impactos.

Por lo general, la información obtenida deberá estar disponible a nivel del programa. Esta información deberá figurar en los informes anuales de ejecución (véase el apartado d). A mediados del ejercicio, deberá estar disponible un resumen de esta información anual, junto con las primeras estimaciones de eficacia (por ejemplo en cuanto a realizaciones y resultados).

\section{d. Informes anuales de ejecución e informe final}

\section{Recuadro 10 Informe anual e informe final de ejecución}

"Cuando se trate de una intervención plurianual, la autoridad de gestión enviará a la Comisión (...) un informe anual de ejecución. A más tardar dentro de los seis meses siguientes a la fecha limite de aplicabilidad de las subvenciones (...) se presentará un informe final a la Comisión."

(...) "Los informes de ejecución y los informes finales incluirán los elementos siguientes: a) cualquier cambio en las condiciones generales que afecte a la ejecución de la intervención, en particular las tendencias socioeconómicas significativas, los cambios en las politicas nacionales, regionales o sectoriales (...), y en su caso de las repercusiones de ello en la coherencia entre las intervenciones de los distintos Fondos o entre éstas y las de otros instrumentos financieros; b) la situación en que se encuentre la aplicación de los ejes prioritarios y las medidas correspondientes a cada Fondo, en relación con sus objetivos específicos, cuantificando (...) los indicadores fisicos, de resultado y de impacto (...); c) la ejecución financiera de la intervención (...), cuantificando los indicadores financieros (...)".

Fuente: Artículo 37 del Reglamento por el que se establecen disposiciones generales sobre los Fondos Estructurales (CE 1260/99).

En el caso de todas las intervenciones plurianuales, la autoridad de gestión designada por el Estado miembro presentará un informe de ejecución a la Comisión Europea dentro de los seis primeros meses siguientes al final de cada año de ejecución. En el informe se presentarán de forma pormenorizada los progresos de la ejecución de la intervención durante el año anterior.

Los Estados miembros elaborarán estos informes sobre la base de los siguientes elementos:

- datos sobre el contexto de ejecución de la intervención;

- progresos realizados en la consecución de las prioridades y objetivos específicos de las medidas y, en su caso, los progresos efectuados en los grandes proyectos, demostrados cuantitativamente mediante los indicadores de seguimiento adoptados al efecto; $y$,
- ejecución financiera de la intervención a nivel de la medida, sobre la base de indicadores cuantificados.

\section{e. Utilización de los indicadores para la evaluación}

Recuadro 11
Utilización de los indicadores de seguimiento
para las evaluaciones
"Con el fin de valorar la eficacia de las intervenciones estructurales, la
acción comunitaria será objeto de una evaluación previa, de una evaluación
intermedia y de una evaluación posterior destinadas a apreciar su impacto
en relación con los objetivos (...) y a analizar su incidencia en problemas
estructurales especificos."
(...) "Las autoridades competentes de los Estados miembros y de la Comisión
se dotarán de los medios convenientes y reunirán los datos necesarios para
que la evaluación pueda efectuarse de la manera más eficaz. La evaluación
utilizará (...) los distintos elementos que pueda proporcionar el sistema
de seguimiento, completados en caso necesario por la recopilación de
informaciones destinadas a aumentar su pertinencia".
"Por iniciativa de los Estados miembros o de la Comisión (...) podrán
realizarse evaluaciones complementarias, si procede temáticas, con el fin
de definir experiencias transferibles".

Fuente: Artículo 40 del Reglamento por el que se establecen disposiciones generales sobre los Fondos Estructurales (CE 1260/99).

Como ya se mencionó, el trabajo de evaluación de cada programa puede dividirse en tres fases principales: evaluación previa, intermedia y posterior. Cada una de estas fases debe referirse a una serie de cuestiones específicas relativas al rendimiento de los programas. Los indicadores de seguimiento representan, pues, una fuente fundamental de información, en la que debe basarse la evaluación.

La evaluación de los impactos (en términos de objetivos globales) se facilitará si los sistemas de seguimiento proporcionan información adecuada sobre los progresos realizados y los resultados obtenidos (por ejemplo, número de personas formadas o número de explotaciones agrícolas con mejoras en los caminos de acceso).

Sin embargo, las evaluaciones pueden utilizar otras metodologías y fuentes de datos, de acuerdo con los objetivos de la investigación, el presupuesto y el tiempo disponibles.

\subsection{Transmisión electrónica de la información}

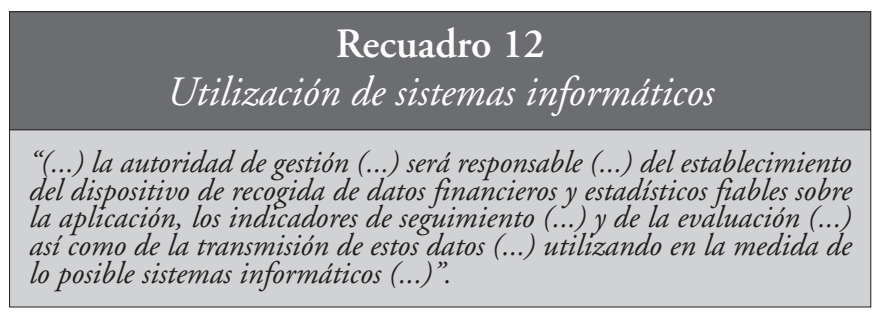

Fuente: Artículo 34 del Reglamento por el que se establecen disposiciones generales sobre los Fondos Estructurales (CE 1260/99). 
En la actualidad es evidente que la informatización de la información facilita las tareas de gestión, seguimiento y evaluación. Por ello, dada la situación de desarrollo de la informática, es útil y oportuno que el sistema de seguimiento esté apoyado en un programa informático en red para la recogida y tratamiento de los datos. Además, es recomendable el diseño de un sistema de flujos de información entre organismos gestores y organismos de coordinación, para la actualización y el mantenimiento de los datos.

\subsection{Elementos clave para la puesta en práctica del Sistema de seguimiento continuo}

En los apartados anteriores se ha argumentado la necesidad y utilidad de la aplicación de un Sistema de seguimiento continuo y la teoría que lo sustenta. La experiencia ha demostrado que los principales problemas que han surgido para la puesta en marcha del seguimiento de programas se han debido sobre todo, a las siguientes cuestiones: (a) confusión entre el seguimiento y la evaluación; (b) falta de información homogénea entre gestores que llevan a cabo actuaciones similares; y (c) escasez de medios informáticos (equipos, software y conexión a Internet) y formación para el almacenamiento, procesamiento y transmisión de la información.

Por ello, a continuación se resumen de forma esquemática los elementos clave que se consideran necesarios para la puesta en práctica y funcionamiento de un Sistema de seguimiento de las actuaciones cofinanciadas con Fondos Europeos.

\section{Recuadro 13}

Condiciones necesarias para establecer y poner en

funcionamiento un sistema de seguimiento continuo

Para poder aplicar un modelo de seguimiento continuo de las acciones comunitarias tal como se presenta en este artículo es necesario:

- Identificar las actividades que se han de realizar o se están realizando.

- Definir los indicadores del programa (de recursos, de realización, de resultados y de impactos).

- Distinguir entre los indicadores de seguimiento (en general de recursos, realización y resultados), es decir, los que serán responsabilidad de los organismos gestores, y los indicadores que se utilizarán en las evaluaciones o en estudios especificos (en general de impacto).

- Seleccionar indicadores de seguimiento que: (1) estén relacionados de forma directa con las actividades concretas que llevan a cabo los organismos gestores; (2) se adecuen al tipo de información que los organismos gestores pueden recoger o elaborar en su actividad diaria; (3) no sean ambiguos; y (4) no sean muy numerosos.

- Prever la recogida de las informaciones relativas a los progresos en la gestión de cada acción. La recogida de información para el seguimiento debe realizarse de manera permanente.

- Seleccionar indicadores del contexto que sean relevantes para definir la situación de partida y para contextualizar las actuaciones que se están llevando a cabo, y responsabilizar a un organismo para la actualización de estos indicadores de forma periódica.

- Asegurar los medios y recursos necesarios (equipos, acceso a Internet y formación) a los coordinadores y a cada uno de los gestores de las acciones cofinanciadas con Fondos Europeos para que puedan recoger, informatizar y transmitir los datos requeridos.

\section{El uso de las evaluaciones}

Desde hace muchos años, tanto los evaluadores profesionales como los responsables públicos que encargan u obligan a hacer evaluaciones, están preocupados por el uso de esas evaluaciones, una vez la investigación ha finalizado (PATTON, 2001: 329). El gasto en la realización de una evaluación no estaría justificado si esa evaluación no lleva a la mejora de la actuación pública evaluada o de actuaciones futuras, es decir, si no consigue mejorar la eficacia y la eficiencia de las Administraciones públicas. El valor de una evaluación no depende de su coste, sino de su uso (Feinstein, 2002).

En el año 2002, la Comisión Europea encargó un estudio al Instituto Tavistock y al Technopolis Francia para analizar el uso de las evaluaciones en los servicios de la Comisión (WiLliams DE LAAT y STERN, 2002). El objetivo principal del estudio era analizar qué mecanismos y condiciones apoyan (o disuaden) la retroalimentación de los resultados de las evaluaciones en la programación e implementación de los programas de la Unión Europea. Según este informe, la utilización de las evaluaciones es, en algunos casos, directa y fácilmente observable, por ejemplo cuando las recomendaciones del evaluador son transformadas en acciones que modifican (o incluso eliminan) programas o servicios públicos. Sin embargo, en la mayoría de las ocasiones, el uso de las evaluaciones es más sutil, con una relación causal entre los resultados de las evaluaciones y los cambios en las intervenciones públicas difícil de identificar. Los cambios pueden surgir indirectamente o después de un considerable lapso de tiempo, de forma acumulativa. Incluso cuando los resultados de una evaluación no son utilizados o son utilizados sólo indirectamente, el proceso de la evaluación, que incluye discusiones y reflexiones, puede ser útil, ya que ofrece oportunidades de intercambiar información y clarificar ideas.

Según este estudio, el grado de utilización de las evaluaciones de los programas de la Unión Europea no depende tanto de la calidad de una evaluación concreta o de la forma en que ésta se ha llevado a cabo, sino de otros factores. Éstos se pueden resumir de la forma siguiente: (i) el factor organizativo (es más efectivo si la unidad responsable de evaluación está separada de las funciones de control, auditoría e inspección); (ii) la forma en que se distribuyen entre los potenciales usuarios y los interesados en general los resultados y recomendaciones de las evaluaciones; (iii) el tipo de relaciones entre las personas responsables de las evaluaciones y las responsables de los programas; y (iv) la existencia de una "cultura de la evaluación" (entendida como la apertura a actitudes de reflexión y autocrítica) en la organización y la institucionalización de sistemas de gestión del conocimiento (Williams, De LaAT y Stern, 2002).

La diferente literatura sobre el tema (recogida por diversas asociaciones de evaluadores como "estándares" para la evalua- 
ción de programas), concluye que, en general, las condiciones que favorecen que las evaluaciones se ajusten a las necesidades de sus potenciales usuarios son las siguientes (BOYLE y LEMAIRE, eds., 1999; Caracelli y Preskill, eds., 2000; ForsS, Rebien y Carlsoon, 2002; Pawson y Tilley, 2001; ValovirTa, 2002; VAN DER KNAPP, 1995; WEISS, 1999):

- Tener claro los diferentes actores, para asegurar que sus necesidades son tenidas en cuenta.

- Asegurar la credibilidad de los evaluadores, para que sea más probable que sus conclusiones sean aceptadas.

- Recoger información relevante de un amplio abanico de fuentes, consultando a los clientes y a los actores implicados.

- Ser claro en los criterios de juicio utilizados para interpretar la información.

- Ser claro en los informes, para que la información contenida en los mismos sea fácil de entender.

- Asegurarse de que los informes son repartidos en un corto espacio de tiempo entre los potenciales usuarios.

- Planificar las evaluaciones desde el principio de forma que estimule a los actores implicados a que lleven a cabo las recomendaciones.
* Este trabajo forma parte de un proyecto de investigación financiado por la Dirección General de Cooperación con el Estado y Asuntos Europeos de la Comunidad de Madrid y los Fondos Estructurales de la Unión Europea.

** Doctora en Ciencias Políticas y Sociología y Master en Administración Pública. Profesora en el Departamento de Ciencia Política y Sociología de la Universidad Carlos III de Madrid y consultora especialista en análisis y evaluación de programas y servicios públicos.
1 Sin embargo, en algunos casos, estas evaluaciones se realizan un año o unos meses antes de la finalización del programa, para disponer de sus conclusiones a la hora de elaborar la programación de los años siguientes. En estas circunstancias, la evaluación suele denominarse "evaluación intermedia avanzada". Si, por el contrario, la evaluación se lleva a cabo dos o tres años después de haber finalizado el período de programación, con el objetivo de dar tiempo a que se materialicen los impactos y poder observar su perdurabilidad, la investigación suele denominarse evaluación ex post.

\section{Bibliografia}

Boyle, Richard y Donald LemarRe (eds.) (1999): Building Effective Evaluation Capacity: Lessons from Practice, New Brunswick: Transaction Publishers.

Caracelli, Valerie J. y Hallie Preskill (eds.) (2000): The Expanding Scope of Evaluation Use, San Francisco: Jossey-Bass.

COMISIÓN EUROPEA (1995), Orientaciones para el seguimiento y las evaluaciones intermedias de los MCA, DOCUP e intervenciones, 34 pág.

COMISIÓN EuROPEA (1999a), Evaluación de los programas de desarrollo rural 2000-2006 financiados con cargo al Fondo Europeo de Orientación y Garantía Agricola. Directrices, Dirección General de Agricultura, 48 pág.

COMISIÓN EUROPEA (1999b), Indicadores de seguimiento y evaluación: orientaciones metodológicas, El nuevo período de programación 2000-2006: Documentos de trabajo metodológicos, Documento de Trabajo 3, Dirección General XVI, Política Regional y Cohesión, Coordinación y Evaluación de Operaciones, 67 pág. (Internet).

COMISIÓN EUROPEA (1999c), La Evaluación previa de las intervenciones en el período 2000-2006, El nuevo período de programación 2000-2006: Documentos de trabajo metodológicos, Documento de trabajo 2, Dirección General XVI, Política Regional y Cohesión, Coordinación y Evaluación de Operaciones, 41 págs.

COMisión Europea (2000), Las acciones estructurales 2000-2006. Comentariosy reglamentos, Luxemburgo, Oficina de publicaciones oficiales de las Comunidades Europeas, 158 págs.

Feinstein, Osvaldo N. (2002): "Use of Evaluations and the Evaluation of their Use”, Evaluation, vol. 8, no 4, págs. 433/439.
Forss, Kim, Claus C. Rebein y Jerker Carlsson (2002): "Process Use of Evaluations: Types of Use that Precede Lessons Learned and Feedback", Evaluation, vol. $8, n^{\circ} 1$, págs. 29/45.

LuUKKONEn, Terttu (2002): "Research evaluation in Europe: state of the art", Research Evaluation, vol. 11, nº 2, pág. 81/84.

PATTON, Michael Quinn (2001): "Evaluation, Knowledge Management, Best Practices, and High Quality Lessons Learned”, American Journal of Evaluation, vol. 22, nº 3, págs. 329/336.

Patton, Michael Quinn (2002): “Weiss' Call for Humility: Further Reflections”, American Journal of Evaluation, vol. 23, nº 2, págs. 231/233.

PaWson, Ray y Nick Tilley (2001): "Realistic Evaluation Bloodlines", American Journal of Evaluation, vol. 22, no 3, págs. 317/324.

Valovirta, Ville (2002): "Evaluation Utilization as Argumentation", Evaluation, vol. 8, nº 1, págs. 60/80.

VAN DER KNAPP, P. (1995): "Policy Evaluation and Learning: Feedback, Enlightenment or Argumentation”, Evaluation, vol. 1, nº 2, págs. 189/216.

WeIss, Carol H. (1999): "The Interface between Evaluation and Public Policy", Evaluation, Vol. 5, n 4, págs. 468/486.

WeIss, Carol H. (2002): "Patton's (2001) 'Lesson's Learned': A Comment", American Journal of Evaluation, vol. 23, nº 2, págs. 229/230.

Williams, Kevin, Baastian de LAAT y Elliot STERN (2002): The Use of Evaluation in the Commission Services, Technopilis France, 56 págs. + anexos (mimeo). 
